\title{
Application of the open operating system 'MindSphere' in digital transformation of high-tech enterprises
}

\section{Применение открытой операционной системы «MindSphere» в цифровой трансформации высокотехнологичных предприятий}

Received: February 20, $2019 \quad$ Accepted: March 22, 2019

\author{
Written by: \\ Sergey V. Novikov ${ }^{4}$ \\ https://orcid.org/0000-0001-6921-1760 \\ https://www.scopus.com/authid/detail.uri?authorId=57192318711 \\ elibrary.ru: https://elibrary.ru/author_profile.asp?id=807011 \\ Andrey A. Sazonov \\ elibrary.ru: https://elibrary.ru/author_profile.asp?id=741927
}

\begin{abstract}
The article is devoted to the peculiarities of the open operating system (OS) application MindSphere in digital transformation of high-tech enterprises. The theoretical part of the article notes that nowadays digital transformation already has a huge impact on various aspects of business, such as strategy, partner management, production, pricing, sales, promotion, and organizational structure. The main task of the MindSphere platform is to provide fast and costeffective connection of the equipment fleet, data collection, and analysis in order to obtain significant production and business results. The MindSphere platform integrates all data sources: Product Lifecycle Management (PLM), Enterprise Resource Planning (ERP), Manufacturing Execution Systems (MES), Quality Management Systems (QMS), Customer Relationship Management (CRM) and Internet of Things (IOT) into one analysis center. In the course of the analysis, the authors found that the MindSphere OS allows for the effective unification, search, filtering and evaluation of data taking into account the context, which gives a significant synergetic effect and provides a basis for intelligent decision-making, and therefore significantly reduces the cost and time to find sources of problems, allowing to focus on optimizing products, production and service, considering the creation of value and increase customer satisfaction. The authors point out that the implementation of IOT solutions opens up a new dimension for business owners in the view of
\end{abstract}

\begin{abstract}
Аннотация
Статья посвящена особенностям применения открытой операционной системы MindSphere в цифровой трансформации высокотехнологичных предприятий. В теоретической части статьи отмечается, что в настоящее время цифровая трансформация уже оказывает огромное влияние на различные аспекты бизнеса, такие как стратегия, управление партнерами, производство, ценообразование, продажи, продвижение и оргструктура. Определена основная задача платформы MindSphere, которая заключается в обеспечении быстрого и экономически эффективного подключения парка оборудования, сбора и анализа данных с целью получения значимых для производства и бизнеса результатов. Платформа MindSphere объединяет все источники данных: управление жизненным циклом продукта (PLM), планирование ресурсов предприятия (ERP), системы управления производством (MES), системы управления качеством (QMS), управление взаимоотношениями с клиентами (CRM) и IOT в один аналитический центр. В ходе проведенного анализа авторами было установлено, что операционная система MindSphere позволяет эффективным образом осуществлять унификацию, поиск, фильтрацию и анализ данных с учетом контекста, что дает значительный
\end{abstract}

\footnotetext{
${ }^{4} \mathrm{PhD}$ in Economics, Associate Professor, Head of Institute of Engineering Economics and Humanities, Moscow Aviation Institute (National Research University), Moscow, Russia.

${ }^{5} \mathrm{PhD}$ in Economics, Associate Professor, Moscow Aviation Institute (National Research University), Moscow, Russia.
} 
their company based on data, and for the company itself provides previously inaccessible speed of operation, transparency of processes and their optimization, also raises the safety of work and other aspects of activity to a new level. In the final part of the article the authors conclude that MindSphere platform has all necessary qualities to be among the leaders of the Industrial IOT (IIOT), to provide customers with the opportunity to qualitatively transform IOT data into useful business results.

Keywords: advanced IT solutions, digital platforms, digital transformation of production, improving business processes, industrial Internet.

\section{Introduction}

Maintaining competitiveness in modern production requires companies not only to produce the highest quality products, but also to maximize operational efficiency in global value chains. One of the main factors exerting pressure on profitability in various industries is unplanned downtime and equipment failures, leading to the shutdown of critical segments of production. Most often, these problems are due to a lack of transparency in the performance and modes of operation of machines that do not allow for predicting and preventing system failures. According to recent research, only 5\% of all available industry data is used by enterprises to improve operational efficiency. Digital transformation already has a huge impact on various aspects of business, such as strategy, partner management, production, pricing, sales, promotion and organizational structure. Coordination in design and production, product and production data analytics, and improvement of after-sales services should become permanent in the future as a guarantee of business sustainability and efficiency.

To make the most of the wealth of data generated by smart technology, a digital twin of the product creation chain (a virtual copy of its lifecycle) is синергетический эффект и обеспечивает базу для интеллектуального принятия решений и следовательно значительно снижает затраты и время на поиск источников проблем, позволяет сосредоточиться на оптимизации изделий, производства и обслуживания с учетом создания стоимости и повышения удовлетворенности клиентов. Авторы отмечают, что внедрение решений IOT открывает владельцам бизнеса новое измерение во взгляде на их компанию, основанную на данных, а для самой компании обеспечивает недоступную ранее скорость операционной деятельности, прозрачность процессов и их оптимизацию, также на новый уровень поднимается безопасность труда и другие аспекты деятельности. В заключение статьи авторы приходят к выводам, что платформа MindSphere имеет все необходимые качества, чтобы быть в числе лидеров индустриального интернета вещей (IIOT), обеспечить возможность заказчикам качественным образом трансформировать IOTданные в полезные бизнес-результаты.

Ключевые слова: передовые ИТ-решения, промышленный интернет, улучшение бизнеспроцессов, цифровая трансформация производства, цифровые платформы.

needed. It is necessary to collect in real time and to analyze the reliable data from already established park of the industrial equipment, control systems of technological processes, logistical and PLM, ERP, MES and Manufacturing Operations Management (MOM) systems. Extracting, collecting, and intelligently analyzing this data in order to develop and make optimal management decisions in a timely manner is a critical success factor in the transformation to a flexible and digital production model.

\section{Theoretical framework}

In the production and service divisions of the Siemens Group, approaches, technologies, methods of production data collection, and analysis have been developed for many years to optimize costs, increase flexibility and robotization of production, and improve product design and maintenance. Various mathematical models of predictive analysis of equipment operation, industrial knowledge bases, algorithms of supply and sales analysis, management and optimization of production chains were created and applied at various "digital" enterprises (Snowden, 2002). 
MindSphere OS is designed to support a wider range of industrial enterprises on the path of digital transformation, offering them a platform for the development and integration of their own applications and services, which will contribute to the further development of innovations in the field of IOT. Software AG has implemented scalable components of its digital business platform into the MindSphere OS to provide customers with the flexibility to manage networks of millions of end devices. The open MindSphere Platform as a Service (PaaS) OS enables the development of an extensive ecosystem of partners and the delivery of new applications that drive new business models, such as preventive maintenance, energy data management and resource optimization. Easy access to the OS is especially important because it allows the use of Application Programming Interface (API) for the development of Original Equipment Manufacturer (OEM) products and applications on request, as well as open connectivity standards (such as Object Linking and Embedding for Process Control Unified Architecture (OPC UA)). Thus, the MindSphere OS forms the basis for new business models (Abdikeev, Bogachev, Bekulova, 2019).

The main task of the MindSphere platform is to provide fast and cost-effective connection of the equipment fleet, data collection and analysis in order to obtain significant production and business results. Companies can use MindSphere to create closedloop production, to seamlessly integrate operational data across the value chain. The MindSphere architecture has several levels (Kurtz, Snowden, 2003). The cloud platform itself is central, providing all the services and interfaces required to run the application layer developed by Siemens partners, customers, and companies. The data collection layer allows connecting different data sources from motors and industrial controllers to different enterprise IT systems and external cloud services. All these layers are "threaded" onto the identification and protection system, in particular, all data transmitted to the platform is protected with Secure Sockets Layer (SSL)/ Transport Layer Security (TLS) encryption with a key length of 256 bits. The MindSphere platform layer provides a secure data storage and a single authentication and access gateway to MindSphere Gateway data. In general, information security issues at all levels of MindSphere are given priority, and cyber security solutions used in MindSphere are based on international standards ISO 27001, IEC 62443, etc., which provides the highest level of protection.

An important feature of the platform layer based on the open source solution Cloud Foundry is the ability to work in different cloud environments and resource virtualization infrastructures. Currently, MindSphere platform is available in Infrastructure as a Service (IaaS)-cloud Amazon Web Services, and in the nearest future the manufacturer plans to provide MindSphere in Microsoft Azure and further in other cloud environments. The MindConnect data collection layer is designed for fast and seamless integration with existing data sources, IT systems and devices from both Siemens (e.g. Simatic S71500 Programmable Logic Controller (PLC) or Sinumerik Numerical Control 840D sl) and other vendors. This can be done using ready-made hardware gateways MindConnect Nano and MindConnect IoT2040, supporting open protocols S7 and OPC UA. To connect the devices can be used open source library MindConnect Lib, which allows you to embed software transfer agents in the platform MindSphere in almost any equipment equipped with a microprocessor and hardware interface coupling (Isaychenkova, 2019).

Production equipment and machines can create large data streams and signals generated with high frequency. The analysis of these signals allows to control and predict the operation of the equipment by comparing the data of the actually functioning system with its digital model, to detect anomalies and deviations. In many cases, it is inefficient and expensive to completely transfer "raw" data to the IOT cloud platform in terms of bandwidth. To meet the challenges of signal processing and streaming data, the MindSphere platform offers additional onsite monitoring and analysis tools. The Condition Monitoring System (CMS) X Tools subsystem is a CMS from Siemens, which includes libraries and signal analysis tools. The integration of CMS X Tools can enrich a MindSphere-based solution with effective spectral and vibration analysis capabilities for signals up to $192 \mathrm{kHz}$, dynamic correlation of data flows, search for anomalies and trend detection (Siemens PLM Software, 2018).

Pre-processed and compressed data are then transferred to the MindSphere cloud platform for further analysis, for example, in the context of related equipment and its history. CMS X Tools software components do not require any specialized hardware and can be installed on industrial computers manufactured by Siemens or on any standard computer or server running Windows. The solution supports a wide range of quick-connect devices that do not require additional modules for integration with the MindSphere platform (Dmitriev, Novikov, 2019). 


\section{Methodology}

As research methods, an analytical assessment of the forecasts of the development of technologies for building and developing digital enterprises presented by experts of the company Siemens PLM Software is used. The study is based on a comprehensive analysis and subsequent assessment of the main results of the implementation of the technological transformation processes of industry, with the subsequent determination of its key areas and directions. The analysis is based on materials from domestic and foreign scientists, data provided by leading high-tech enterprises. Now we are going to consider the components of the platform MindSphere (Skripkin, 2017):

\section{- Research and visualization:}

- Tableau-based data research, analytics and visualization tools;

- search for problem points;

$\circ$ trend analysis and search for optimal solutions.

\section{- Process creation:}

- creation of processing processes, data enrichment, and response to events based on Node-RED;

- processes started manually, based on temporary events (or thresholds) or through the RESTful API.

- Flow analysis:

- quickly customizable application for analyzing streams/series from devices;

- quick analysis and display of data for an effective understanding of the installed fleet;

$\circ$ transferring results to customers and partners.

\section{- Report building:}

- creating individual reports and data display panels based on TIBCO Jaspersoft solution;

- combination of various data sources, search for dependencies and relationships in data;

- Modeling:

○ building predictive models, including machine learning;

- various algorithms and libraries, including TensorFlow, Spark MLlib, NumPy, Scikit Learn, Keras, SciPy, Matplotlib, Pandas, Theano Hi Pengcheng

- The MindSphere platform combines all data sources: PLM, ERP, MES, QMS, CRM, and IOT into one analytical center. MindSphere OS allows (Ananyin, Zimin, Lugachev, Gimranov, Skriprin, 2018):

$\circ$ search and analyze data from suppliers, manufacturers, and customers within seconds;
- shows a complete picture of the entire value chain;

- makes it possible to close the chains between the design, production, and operation of the product;

o provides tools for quick contextual search, data quality analysis, performance analysis, and visualization.

Consequently, MindSphere OS allows efficient unification, search, filtering, and analysis of data taking into account the context, which gives a significant synergistic effect and provides the basis for intelligent decision making. This significantly reduces the cost and time of searching for sources of problems, allows focusing on optimizing products, production, and services, taking into account value creation and increasing customer satisfaction (Dmitriev, Novikov, 2019).

Using web-based tools for graphical configuration of connections, in the context of MindSphere, we can create flexible integration that integrates enterprise systems both in the cloud and locally.

MindConnect API. It is an API that allows the customer to both program and use special connectors that interact with the standard MindSphere API.

MindConnect Integration. It is an option to connect multiple data management systems to MindSphere, including ERP, MES, and Supervisory Control and Data Acquisition (SCADA) systems.

MindAccess Developer Plan. The developer's plan provides the creation of reliable IOT applications using in-depth analytics and services, including data management, intelligent training and visualization, to accelerate the development process. Developers can easily make, register, and test their applications, as well as access to an ever-expanding list of API and services.

MindAccess Operator Plan. An Operator Plan that provides a dedicated environment for launching production applications. System administrators can seamlessly manage, run, track, and publish applications for customers and partners using the MindSphere Store.

Digitalization of production involves change at all levels (Shamaeva, 2018):

- Business level:

- new design thinking and focus on customers;

- active ecosystem (open innovation, extended enterprise, and data sharing); 
- modular structure of products and services;

- innovative culture, flexibility, and risk orientation.

- Operational level:

- transformations in design, production, marketing, sales, and service;

- new competencies and roles (selling new values, data analytics, and new services);

o changes in organizational structure (new knowledge, new culture, customer experience, and shared responsibility).

- Technological level:

- focus on scalability, modularity, and reuse; - lifecycle data analytics;

○ data infrastructure and information security;

- flexibility of operations and minimization of operational risks.

Neither IOT project can be divorced from the specifics of the client's company business, that is why none of the technical or organizational decisions can be made without mutual preparatory work. The result of this work is a business plan for the implementation of IOT solutions. A business plan is only a hypothesis about how effective the solution will actually be, and this hypothesis is tested at the mandatory stage of pilot implementation on small volumes (short term, small number of physical objects, etc.). As a rule, the cost of such a "pilot" is much lower than full-scale implementation and future benefits and justifies its implementation. The general approach to solving problems using IOT includes the following (Isaychenkova, 2019):

- data collection, accumulation, and analysis. This stage can be shortened if there is already accumulated data and experience in the subject area.

- creating and linking models. It includes verification of models and determination of the basic boundary conditions for their functioning.

- definition of general rules and automation. There are response rules, including automation of decision-making processes.

Implementing IOT solutions opens up a new dimension for business owners in looking at their data-driven company. For the company itself, it provides the previously inaccessible speed of operations, transparency of processes and their optimization. Work safety and other aspects of activity are also rising to a new level. At the stage of technical implementation, specialists perform the following (Kurtz, Snowden, 2003):
- development of network and software architecture solutions;

- preparation of IT infrastructure, if necessary, work can be carried out both with client capacities and in cloud or hybrid hosting;

- development of code for client and server applications, as well as embedded software;

- functional, integration and load testing;

- development of documentation.

Now we are going to consider the value of digital transformation of your existing IT infrastructure with MindSphere:

- Equipment manufacturer:

- increase of efficiency/reduction of costs for warranty support;

○ visualization and control of the established fleet;

automatic notification of failures and accidents;

- new business models and services;

- directed provision of a guaranteed level of reliability;

○ product improvement through feedback and job analysis;

- use of operational data for validation, model optimization, and engineering.

- Equipment operator:

○ increase productivity/equipment uptime;

○ predicting failures for preventive maintenance, reducing downtime or unplanned outages;

○ fleet optimization;

optimal configuration and maximum load of equipment, peak analysis, energy consumption, etc.;

$\circ$ improving the efficiency of equipment maintenance;

- lengthening of service cycles due to optimal performance.

\section{Results}

The tasks of production optimization, of course, not only include monitoring and diagnostics of industrial equipment. It is necessary to promptly consider the condition of the warehouse, information from suppliers and customers, quality parameters of raw materials and products, etc. MindSphere Product Intelligence application includes all necessary interfaces and tools for data collection and analysis from different sources (suppliers, manufacturers, and customers) in combination with industrial data. The solution allows for the unification, search, filtering, and analysis of data according to the context (e.g. product batch, component supply, 
production conditions, and operating conditions). This enables intelligent decision making and reduces costs and time to source problems (Kurtz, Snowden, 2003).

Regardless of their purpose, the key characteristics of IIOT-platforms and applications that fundamentally distinguish them from "traditional" means of automation, which can include almost all domestic developments of them, are as follows (Novikov, Veas Iniesta, 2019).

Cloud (public, hybrid) model of providing application functions, which, unlike the model of selling licenses for on-premise installations allows provider/developer to accumulate and analyze data of all connected users of the application. The array of data and its analysis models are the main assets of any developer of such applications, which are used by strategic investors when estimating the developer's value.

Openness of the platforms and services provide intensive two-way information exchange with a wide variety of external systems. API-integration with ERP and accounting applications, with PLM/SLM applications, systems of suppliers, and buyers with implementation of through traceability is obligatory.

Full functionality 6 which occurs at the expense of formation of the ecosystems consisting of "base" and analytical IIOT-platforms, applications IOT ERP and IOT PLM, total functionality of such IIOTecosystems covers business processes of the account and planning of the top level (ERP, Business Intelligence (BI)), and Automated process control system (Automated Process Control System (APCS)), and also processes of management of products and services on all their lifecycle (PLM/SLM).

Many Siemens companies have already carried out a deep integration of MindSphere analytics into endto-end production and product design processes. The factory of industrial controllers of family Simatic by means of analytics and data exchange between cloud platform MindSphere, MES-system Simatic IT, PLM-system Teamcenter and ERP-system SAP could reach $75 \%$ automation of processes of manufacture for more than 1000 variants of products. Depending on the orders placed by customers, the plant automatically reconstructs production lines up to 350 times a day (Snowden, 2002). Optimization of equipment and processes loading allowed increasing production volume nine times since the beginning of production at the same areas and the same number of staff. Thanks to adaptive quality control of technological processes and components, the output of suitable products reaches $99.85 \%$

\section{Conclusions}

Thanks to the rapid development of information and communication technologies, as well as the steadily growing demand for IOT solutions, dozens of IOT platforms enter the market every year. At the same time, previous experience in platform development in such areas as social networks, transport, hospitality, and other segments shows that as a result of competitive selection and subsequent consolidation, three to five platforms, both with technical advantages and offering profitable and efficient business models, are reaching the finish line. The MindSphere platform is a global partner ecosystem that provides sustainable IOT solutions and flexible services to meet customer requirements. MindSphere offers partners an unprecedented opportunity to participate in the digital transformation of companies, regardless of industry or size. Customers can optimize the entire value chain from design and manufacturing to productivity.

The unpreparedness of Russian industrial enterprises for a cardinal digital transformation is the main constraint to the development of IIOT-platforms and applications ecosystems in Russia. In this regard, it is important for Russian developers of IIOTplatforms and applications to understand that the currently forming global ecosystem of cloud-based IIOT applications, each of which performs its role and interacts with others, is still open to new players.

Using web-based tools for graphical configuration of connections, it is possible in the context of MindSphere to create a flexible integration that combines corporate systems, both in the cloud and locally, which means that the MindSphere platform has all the necessary qualities to be among the leaders of the IIOT, providing customers with the ability to transform IOT data into useful business results. At the same time, the platform stimulates the development of a wide ecosystem of partners who build their business on innovative digital services. Available in Russia MindSphere platform now opens new opportunities of the digital age also for Russian customers and partners.

\section{References}

Abdikeev N.M., Bogachev Yu.S., Bekulova S.R. (2019). Institutional mechanisms for ensuring a scientific and technological breakthrough in the Russian economy. Management Sciences. 9 (1), 619. 
Ananyin V.I., Zimin K.V., Lugachev M.I, Gimranov R.D., Skriprin K.G. (2018) Digital organization: Transformation into the new reality. Business Informatics. 2(44), 45-54.

Dmitriev O.N., Novikov S.V. (2019). Concept of state management doctrine. Amazonia Investiga. 8(22), 238-246.

Dmitriev O.N., Novikov S.V. (2019). Verification of Feasibility Studies at High-Technology Enterprises. Russian Engineering Research. 39(9), 780-781.

Isaychenkova V.V. (2019). Ensuring the competitiveness of an industrial enterprise in a digital economy. Century of quality. 2, 91-105.

Kurtz C.F., Snowden D.J. (2003). The new dynamics of strategy: Sense-making in a complex and complicated world. IBM Systems Journal. 42(3), 462-483.

Novikov S.V., Veas Iniesta D.S. (2019). Analysis of development trends in the innovation industry of the
Russian Federation. Amazonia Investiga. 8(19), 298307.

Shamaeva N.P. (2018). The increasing role of innovation as a factor in sustainable economic growth. Bulletin of the Udmurt University. Series Economics and Law. 28(6), 785-791.

Siemens PLM Software (2018). MindSphere cloud-based, open-source operating system for the Internet of things, conducive to digital business transformation. CAD/CAM/CAE Observer. 6(114), 68-76.

Skripkin K.G. (2017). How environment influences on organizational design of educational institution: analytical instruments. Modern Information Technologies and IT Education. 12(3), 225-236.

Snowden D. (2002). Complex acts of knowing: Paradox and descriptive self-awareness. Journal of Knowledge Management. 6(2), 100-111. 